\title{
Computational Ghost Imaging for Remote Sensing
}

Ghost imaging is used in encryption, remote sensing, and biomedical imaging applications.

\section{NASA's Jet Propulsion Laboratory, Pasadena, California}

This work relates to the generic problem of remote active imaging; that is, a source illuminates a target of interest and a receiver collects the scattered light off the target to obtain an image. Conventional imaging systems consist of an imaging lens and a high-resolution detector array [e.g., a CCD (charge coupled device) array] to register the image. However, conventional imaging systems for remote sensing require high-quality optics and need to support large detector arrays and associated electronics. This results in suboptimal size, weight, and power consumption.

Computational ghost imaging (CGI) is a computational alternative to this traditional imaging concept that has a very simple receiver structure. In CGI, the transmitter illuminates the target with a modulated light source. A single-pixel (bucket) detector collects the scattered light. Then, via computation (i.e., postprocessing), the receiver can "reconstruct" the image using the knowledge of the modulation that was projected onto the target by the transmitter. This way, one can construct a very simple receiver that, in principle, requires no lens to image a target.

Ghost imaging is a transverse imaging modality that has been receiving much attention owing to a rich inter- connection of novel physical characteristics and novel signal processing algorithms suitable for active computational imaging. The original ghost imaging experiments consisted of two correlated optical beams traversing distinct paths and impinging on two spatially-separated photodetectors: one beam interacts with the target and then illuminates on a single-pixel (bucket) detector that provides no spatial resolution, whereas the other beam traverses an independent path and impinges on a high-resolution camera without any interaction with the target. The term "ghost imaging" was coined soon after the initial experiments were reported, to emphasize the fact that by cross-correlating two photocurrents, one generates an image of the target. In CGI, the measurement obtained from the reference arm (with the high-resolution detector) is replaced by a computational derivation of the measurement-plane intensity profile of the reference-arm beam. The algorithms applied to computational ghost imaging have diversified beyond simple correlation measurements, and now include modern reconstruction algorithms based on compressive sensing.

The physical principles underpinning CGI are as follows: the transmitter, by use of a spatial light modulator, projects a spatiotemporally varying speckle pattern on the target. The scattered light from the target is collected with a simple bucket detector offering no spatial resolution. The photocurrent, whose fluctuations in excess of the shot-noise floor are proportional to the sum of the fluctuations seen in the transmitter-generated speckles, is then processed to resolve the transverse profile of the object. This signal processing can take on a rather elementary linear form such as cross-correlation, or can be more complex and nonlinear, such as L1-norm minimization. The latter form of ghost imaging is known as compressive, as it utilizes techniques developed for compressive imaging. Turbulence near the target has negligible impact on ghost imaging. The most restrictive source of speckle in remote sensing is that induced by the diffuse surface scattering from the target itself. It is evident from earlier analysis that once the speckle is fully developed, no additional gain is possible from integration, and de-correlated speckles must be obtained by using angular, spectral, or polarization diversity.

This work was done by Baris I. Erkmen of Caltech for NASA's Jet Propulsion Laboratory. For more information, contact iaoffice@jpl.nasa.gov. NPO-48157

\section{Digital Architecture for a Trace Gas Sensor Platform}

\section{John H. Glenn Research Center, Cleveland, Ohio}

A digital architecture has been implemented for a trace gas sensor platform, as a companion to standard analog control electronics, which accommodates optical absorption whose fractional absorbance equivalent would result in excess error if assumed to be linear. In cases where the absorption (1-transmission) is not equivalent to the fractional absorbance within a few percent error, it is necessary to ac- commodate the actual measured absorption while reporting the measured concentration of a target analyte with reasonable accuracy. This requires incorporation of programmable intelligence into the sensor platform so that flexible interpretation of the acquired data may be accomplished.

Several different digital component architectures were tested and implemented. Commercial off-the-shelf digi- tal electronics including data acquisition cards (DAQs), complex programmable logic devices (CPLDs), field-programmable gate arrays (FPGAs), and microcontrollers have been used to achieve the desired outcome. The most completely integrated architecture achieved during the project used the CPLD along with a microcontroller. The CPLD provides the initial digital demodulation of the raw sensor signal, 